\title{
Long-Term Outcomes in Patients after Discontinuation of Immune Checkpoint Inhibitors
}

\author{
Zin W. Myint ${ }^{1,2, *}$, Ruta Arays ${ }^{1,2}$, Arun K. Arumugam Raajasekar ${ }^{1,2}$, Peng Wang ${ }^{1,2}$ \\ ${ }^{1}$ Department of Internal Medicine, Division of Medical Oncology, University of Kentucky Medical Center, Lexington, Kentucky \\ ${ }^{2}$ Markey Cancer Center, University of Kentucky, Lexington, Kentucky \\ *Correspondence author: Zin Myint, MD, CC402 Ben F. Roach Building, 800 Rose Street, Lexington, KY 40536; Phone: 859-323-3964; E-mail: zin.myint@uky.edu
}

Received: September 09, 2018; Accepted: September 17, 2018; Published: September 25, 2018;

\begin{abstract}
Introduction: Programmed death protein/ligand 1 (PD1/PDL1) inhibitors are approved for several advanced or metastatic malignancies with improvement in both progression free and overall survival. However, there is a paucity of data on optimal treatment duration. Both KEYNOTE-001 and KEYNOTE-006 studies showed durable antitumor activity in approximately $90 \%$ of melanoma patients after discontinuation of two-year pembrolizumab treatment, with a median follow up duration of 32 and 33.9 months respectively. To our knowledge, there has been no study that provides similar information for other types of solid cancers. Hence, we report a retrospective single institute experience of the durable response after discontinuation of PD1/PDL1 inhibitors.
\end{abstract}

Materials \& Methods: Data on patients treated with immunotherapy between 2010 and 2017 were collected retrospectively. Patients with all types of cancers who achieved disease control (including stable disease, partial and complete response) and were no longer treated with the immunotherapy for any reason were included. We analyzed the outcomes of these patients after discontinuation of immunotherapy.

Results: We evaluated a total 282 patients with a variety of solid tumor types who were treated with PD1/PDL1 inhibitors; 20 patients met our criteria. Cases were divided into two groups; melanoma (total 8), and non-melanoma (total 12; 3 renal cell, 3 bladder, 1 hepatocellular, 1 colon, and 4 non-small cell lung). As of Jan 2018, 7 out of 8 (88\%) in the melanoma group had disease control after a median follow-up of 9 months post-treatment, whereas 8 out of $12(67 \%)$ in non-melanoma group had disease control after a median follow-up of 10 months. The median treatment cycle in the melanoma group was 11.5 cycles (range: 3-20) versus 11 cycles (range: 3-15) in non-melanoma group. The main reason for stopping treatment was patient preference.

Conclusion: Our study shows similar efficacy of durable response in melanoma patients after stopping immunotherapy compared to KEYNOTE studies. Despite limited sample size and short duration of follow-up, our study was the first showing sustained disease control in several non-melanoma cancers after discontinuation of approximately one-year PD1/PDL1 inhibitor treatment. Future prospective malignancy specific trials for optimal duration are warranted.

Keywords: Immunotherapy, long term outcomes, melanoma, non-melanoma

\section{Introduction}

Immune checkpoint inhibitors have transformed the field of oncology. This treatment modality has been approved for several solid tumors with improvement in both progression free and overall survival. Yet, there is a paucity of data with respect to the optimal treatment duration and availability of a biomarker to predict treatment response. Both KEYNOTE-001 and 006 studies showed that 24-month disease free survival rate from time of complete remission was $90 \%$ vs. $55 \%$ after discontinuation of two-year pembrolizumab treatment. To our knowledge, there is no study that reports similar information for other types of solid cancers. As a retrospective single-center experience, we evaluated durable antitumor activity in both melanoma and nonmelanoma patients who achieved treatment response (complete, partial or stable) after discontinuation of immunotherapy for any reason.

\section{Materials \& Methods}

Data on patients treated with immunotherapy from Jan 2010 to Dec 2017 were collected retrospectively. Patients with any type of cancer that achieved a treatment response (complete, partial or stable) and had completed immunotherapy for any reason were included. Patient demographic information including age, sex, primary cancer, prior therapy, total number of doses, and duration of treatment response after discontinuation of therapy and types of response (complete, partial, stable or progression) were obtained. The study approved by local Institutional Review Board (IRB) and the IRB protocol number is 43216 .

\section{Results}

We evaluated a total 282 patients with a variety of stage IV solid cancers who were treated with immune checkpoint inhibitors. Of that 
group, 157 patients were treated with nivolumab and 125 patients were treated with pembrolizumab. In this single-center, retrospective study, 20 patients met our eligibility criteria. This cohort was divided into two groups: a melanoma group (total 8), and a non-melanoma group (total of 12; 3 renal cell, 3 bladder, 1 hepatocellular, 1 colon, and 4 non-small cell lung). Patient baseline characteristics are detailed in

Table 1 .

Table 1. Baseline Characteristics and Outcomes.

\begin{tabular}{|c|c|c|c|c|c|c|c|c|c|}
\hline Case & Age & Sex & Cancer & Previous Therapy & $\begin{array}{c}\text { Total \# Treatments } \\
\text { (doses) }\end{array}$ & $\begin{array}{c}\text { Reason to } \\
\text { Discontinue }\end{array}$ & $\begin{array}{c}\text { Immune-related } \\
\text { AEs }\end{array}$ & $\begin{array}{c}\text { Duration of } \\
\text { Response (mos) }\end{array}$ & Outcome \\
\hline 1 & 60 & $\mathrm{~F}$ & Melanoma & Ipi $\times 4$ & Pem $\times 10$ & $\begin{array}{l}\text { Organizing } \\
\text { pneumonia }\end{array}$ & $\begin{array}{l}\text { Organizing } \\
\text { pneumonia }\end{array}$ & 28 & $\mathrm{CR}$ \\
\hline 2 & 89 & $\mathrm{~F}$ & Melanoma & None & Pem $\times 17$ & Patient Request & None & 9 & $\mathrm{CR}$ \\
\hline 3 & 77 & M & Melanoma & None & Pem $\times 10$ & Patient Request & None & 2.3 & $\mathrm{CR}$ \\
\hline 4 & 67 & M & Melanoma & Interferon; BRAF & Nivo $\times 20$ & RA Exacerbation & RA Exacerbation & 9.1 & CR \\
\hline 5 & 90 & $\mathrm{~F}$ & Melanoma & None & Pem $\times 13$ & Patient Request & None & 5 & $\mathrm{CR}$ \\
\hline 6 & 53 & $\mathrm{~F}$ & Melanoma & BRAF & $\mathrm{Pem} \times 8$ & RA & $\begin{array}{c}\text { Hypothyroid } \\
\text { \& RA }\end{array}$ & 4.6 & CR \\
\hline 7 & 60 & M & Melanoma & No & $\mathrm{Niv} \times 8$ & Pancreatitis & Pancreatitis & 4.8 & SD \\
\hline 8 & 87 & M & Melanoma & Ipi $\times 4$ & $\mathrm{Niv} \times 3$ & Patient Request & None & 16.7 & $\mathrm{CR}$ \\
\hline 9 & 67 & M & $\mathrm{RCC}$ & Pazopanib & Niv $\times 25$ & Patient Request & None & 6 & $\mathrm{P}$ \\
\hline 10 & 40 & M & $\mathrm{RCC}$ & Sunitinib & $\mathrm{Niv} \times 30$ & Patient Request & None & 11.3 & SD \\
\hline 11 & 74 & $\mathrm{~F}$ & $\mathrm{RCC}$ & Pazopanib & $\mathrm{Niv} \times 20$ & Sjogren & Sjogren & 4.2 & SD \\
\hline 12 & 87 & M & Bladder & Cis/Gem & Pem x11 & Patient Request & None & 11 & SD \\
\hline 13 & 58 & M & Bladder & Carbo/Gem & Pem $\times 13$ & Patient Request & None & 10.6 & PR \\
\hline 14 & 70 & M & Bladder & Carbo/Gem & Pemx 12 & HLH & HLH & 12 & $\mathrm{P}$ \\
\hline 15 & 58 & M & $\mathrm{HCC}$ & Embolization & Nivx6 & Patient Request & None & 11 & $\mathrm{P}$ \\
\hline 16 & 79 & $\mathrm{~F}$ & NSCLC & Carbo/pem & Niv $\mathrm{x} 8$ & Hepatitis & Hepatitis & 7 & PR \\
\hline 17 & 78 & M & NSCLC & Carbo/Gem & Nivx 18 & Patient Request & None & 6 & $\mathrm{P}$ \\
\hline 18 & 76 & F & NSCLC & Unknown & $\mathrm{Niv} \times 24$ & Patient Request & None & 14 & $\mathrm{SD}$ \\
\hline 19 & 66 & $\mathrm{~F}$ & NSCLC & None & Pemx 15 & Patient Request & None & 22.6 & $\mathrm{CR}$ \\
\hline 20 & 55 & $\mathrm{~F}$ & Colon & FOLFOX & Pemx9 & $\begin{array}{l}\text { Peripheral } \\
\text { neuropathy }\end{array}$ & $\begin{array}{l}\text { Peripheral } \\
\text { neuropathy }\end{array}$ & 8.8 & PR \\
\hline
\end{tabular}

Ipi = ipilimumab; Pem = pembrolizumab; Niv = nivoluamb; Cis/Gem = cisplatin/gemcitabine; Carbo/Gem = carboplatin/gemcitabine; carbo/pem =carboplatin/pemetrexed; FOLFOX = Folinic acid+Fluorouracil+Oxaliplatin; mos= months; $\mathrm{AEs}=$ adverse responses; $\mathrm{CR}=$ complete response; $\mathrm{P}=$ progression; $\mathrm{SD}=$ stable disease; $\mathrm{PR}=$ partial response; $\mathrm{BLE}=$ bilateral lower extremities; RA = Rheumatic arthritis; HLH = Hemophagocytic lymphohistiocytosis .

In melanoma group, the median age was 73 years (range: 44-90), with male and female equally represented. Performance status (Eastern Cooperative Oncology Group, ECOG) for all patients was 0 to 1. Four of the 8 melanoma patients had prior therapies; two patients received four doses of adjuvant ipilimumab and two patients were treated with BRAF inhibitors. Three of the 8 patients had the BRAFV600E mutation. Five of the eight melanoma patients were treated with pembrolizumab and the remaining three patients were treated with nivolumab. The median duration of the first radiographic response was 5 months (range: $3-8$ ). The median number of immunotherapy treatment doses was 12 (range: 3-20). The median duration of durable treatment response after discontinuation of immunotherapy was 9.7 months (range: 2-27). Four patients discontinued treatment due to immune-related adverse events: 1 developed organizing pneumonia; 2 acquired Rheumatoid arthritis; and 1 developed pancreatitis. The remaining four patients requested to stop treatment. (Tables 1, 2)

In non-melanoma group of 12 patients, three were diagnosed with renal cell cancer (RCC), three with bladder cancer, there was one hepatocellular cancer (HCC), one colon cancer, and four patients with non-small cell lung cancer (NSCLC). The median age was 67 years (range: 40-87). Eight of the twelve were male and 4 were female. Ten of 12 patients had prior therapies. Three patients were treated with tyrosine kinase inhibitors (two with pazopanib and one with sunitinib) for RCC. Three bladder cancer patients received platinum with 
gemcitabine. One HCC had localized therapy by embolization. Two of the 4 NSCLC had chemotherapy (1 with carboplatin/gemcitabine for squamous cell, 1 with carboplatin/pemetrexed for adenocarcinoma). One colon cancer patient was treated with FOLFOX. Three of the 8 patients were positive for the BRAFV600E mutation. Seven patients were treated with nivolumab, and four patients were treated with pembrolizumab. The median duration of the first radiographic response was 3.2 months (range: $2-5$ ). The median number of immunotherapy treatment doses was 15 (range: 6-30). The median duration of durable treatment response after discontinuation of immunotherapy was 10.4 months (range: 4-23). Four patients discontinued treatment due to immune related adverse events: 1 developed Sjogren syndrome; 1 developed hemophagocytic lymphohistiocytosis; 1 acquired hepatitis, and 1 had peripheral neuropathy. The remaining patients requested a break from treatment. Four patients had disease progression after discontinuation of immunotherapies with the median duration of 8.7 months. (Tables 1, 2)

Table 2: Outcome Comparisons for the Melanoma and Non- Melanoma groups.

\begin{tabular}{|c|c|c|}
\hline Characteristics & $\begin{array}{l}\text { Melanoma } \\
\quad \mathbf{N}=\mathbf{8}\end{array}$ & $\begin{array}{l}\text { Non-melanoma } \\
\quad \mathbf{N}=12\end{array}$ \\
\hline Median age in years (range) & $73(44-90)$ & $67(40-87)$ \\
\hline Men, N (\%) & $4(50 \%)$ & $8(66 \%)$ \\
\hline ECOG $0-1, \mathrm{~N}(\%)$ & $8(100 \%)$ & $12(100 \%)$ \\
\hline Previous therapy, N (\%) & $4(50 \%)$ & $10(83 \%)$ \\
\hline BRAF mutant positive & $3(38 \%)$ & 0 \\
\hline PD-L1 positive $(>50 \%)$ & Unknown & 0 \\
\hline $\begin{array}{l}\text { Median duration of first } \\
\text { radiographic response in } \\
\text { months (range) }\end{array}$ & $5(3-8)$ & $3.2(2-5)$ \\
\hline $\begin{array}{l}\text { Median number of } \\
\text { immunotherapy cycles, } \\
\text { (range) }\end{array}$ & $12(3-20)$ & $15(6-30)$ \\
\hline $\begin{array}{l}\text { Median duration in months } \\
\text { of treatment response after } \\
\text { discontinuation of therapy, } \\
\text { (range) }\end{array}$ & $9.7(2-27)$ & $10.4(4-23)$ \\
\hline $\begin{array}{l}\text { Disease response (CR, PR, } \\
\text { stable, progression) }\end{array}$ & $\begin{array}{c}7(88 \%) \mathrm{CR} \\
1(12 \%) \\
\text { stable }\end{array}$ & $\begin{array}{c}3(25 \%) \mathrm{PR} \\
4(33 \%) \text { stable } \\
4(33 \%) \text { progression } \\
1(8 \%) \mathrm{CR}\end{array}$ \\
\hline $\begin{array}{l}\text { Immune related side effects, } \\
\mathrm{N}(\%)\end{array}$ & $4(50 \%)$ & $4(33 \%)$ \\
\hline
\end{tabular}

ECOG $=$ Eastern Cooperative Oncology Group $\mathrm{CR}=$ complete response; $\mathrm{PR}=$ partial response; $\mathrm{N}=$ number.

Seven out of 8 patients (88\%) from the melanoma group had disease free progression after a median follow-up of 9 months. Eight out of 12 patients (67\%) from the non-melanoma group had disease free progression after a median follow-up of 10 months.

\section{Discussion}

Immune checkpoint inhibitors have transformed oncologic therapeutics. Immunotherapy provides improved overall survival and progression free survival for several solid tumors including melanoma, NSCLC, bladder cancer and RCC. However, there is no good biomarker that predicts which patient will benefit with immunotherapy. It was thought that programmed death ligand 1 (PDL1) expression would predict a good prognostic biomarker, however, there are multiple limitations related to this new type of therapeutic [1-3]. The optimal duration for therapy is ambiguous, however the current practice is to continue immunotherapy until disease progression or intolerable adverse events (AEs) occur. Immunotherapy is associated with immune-related AEs, some that can cause toxicities of grade 3 or higher. Thus, a shorter duration of therapy could spare patients from unnecessary toxicities and health expenditures.

Most patients in our study were treated with immunotherapy as a second-line therapy. Approximately $50 \%$ of patients from the melanoma group and 33\% from the non-melanoma group experienced immunerelated AEs, which led to discontinued treatment. The remaining 50\% (melanoma) and 67\% (non-melanoma) of the patients requested a therapy break secondary to a variety of issues. A common issue was financial, related to limitations imposed by insurance companies. Another issue related to transportation difficulties, especially when bi-weekly commutes were required. Finally, some patients questioned why they would need to continue treatment if they had already achieved a complete response. The median duration of treatment in both groups was approximately one-year, and $88 \%$ of the melanoma group, $67 \%$ from non-melanoma group achieved disease control with a median follow-up of 9-10 months.

Schadendorf et al. hypothesized that the immune-related AEs could be a hint for durability in the response, secondary to activation of immune system [4]. The KEYNOTE-006 phase III trial prospectively investigated for a two-year treatment of pembrolizumab in advanced melanoma. The study demonstrated that 55\% of patients on pembrolizumab had a 24 -months overall survival benefit after discontinuation of the treatment [5]. Conclusions from the KEYNOTE-001 phase III trial supported those reported in the KEYNOTE-006 trial, that patients who achieved a complete response could consider a discontinuation of the treatment. In fact, $90.9 \%$ of those patients who discontinued treatment were in disease-freeremission after a median follow up of two years [6].

These two KEYNOTE studies provide convincing evidence that a two-year immunotherapy treatment period improves overall survival benefit. When compared to the KEYNOTE studies, our study provided similar efficacy in terms of a durable response in melanoma patients in the post immune-treatment period. Interestingly, our patient cohort received treatment for approximately one year. A limitation of our study is that it is a retrospective study.

Cancer cells become resistance to chemotherapy and targeted therapies. An understanding of this concept is important in the sequencing of therapeutics for our patients. Well-performed studies established that mutations develop during ontogenesis that can be driver or passenger mutations, and give rise to non-epitopes that the immune system recognizes as neo-antigens. The immune checkpoint inhibitors act on specific tumor mutant proteins to reactivate the T-cell response. Hence, cases with a high tumor mutation burden 
(TMB) have a high treatment response rate [7, 8]. Studies have suggested that a high TMB predicts a favorable treatment response and also durable clinical benefit. For example, a high TMB in NSCLC predicted a higher response rate $59 \%$ vs $12 \%$ in low TMB patients and longer progression free survival (14.5 months in high TMB patients vs. 4.1 months in low TMB) in patients receiving a PD-1 inhibitor [9]. For bladder cancer, a high TMB did predict a favorable response [10]. Treated tumors exposed to genotoxic chemotherapy or radiotherapy are known to have a higher mutational rate compared to the TMB at diagnosis or prior to treatment $[11,12]$. Therefore, TMB is an emerging biomarker of positive response to immune checkpoint inhibitors. However, efforts should be made to develop a molecular profile of prognostic variables or predictive biomarkers to customize prognosis at the baseline, and thereby guide an optimal duration for the immunotherapy.

\section{Conclusion}

Our study showed a sustained durable response in a variety of solid tumors after discontinuation of immunotherapy with a median 9-10 months of disease free progression post-therapy after median 11 cycles of treatment. Additional prospective trials are warranted.

\section{Declaration Statement}

Ethics Approval and Consent to Participate: The study approved by local Institutional Review Board (IRB) and the IRB protocol number is 43216 .

Consent for Publication: Not applicable.

Availability of Data and Material: Data sharing is not applicable to this article as no datasets were generated or analyzed during the current study.

Author Contributions: Conception and design: All authors; Administrative support: PW; Provision of materials: All authors; Collection and assembly of data: All authors; Data interpretation: All authors; Manuscript writing and final approval: All authors

\section{Compliance with Ethical Standards: Not Applicable}

Conflict of Interest: The authors declare that they have no conflict of interest.

\section{Funding: Not Applicable}

Consent for Publication: Not Applicable. The study approved by local institution review board.

Acknowledgements: The authors thank Catherine Anthony, Ph.D. and the Markey Cancer Center Research Communications Office for assistance with manuscript preparation.

\section{References}

1. Rimm DL, Han G, Taube JM, Yi ES, Bridge JA, et al (2017) A Prospective, Multiinstitutional, Pathologist-Based Assessment of 4 Immunohistochemistry Assays for PD-L1 Expression in Non-Small Cell Lung Cancer. JAMA Oncol. 3: 1051-8. [Crossref]

2. McLaughlin J, Han G, Schalper KA, Carvajal-Hausdorf D, Pelekanou V, et al. (2016) Quantitative Assessment of the Heterogeneity of PD-L1 Expression in NonSmall-Cell Lung Cancer. JAMA Oncol. 2: 46-54. [Crossref]
3. Grigg C,Rizvi NA. (2016) PD-L1 biomarker testing for non-small cell lung cancer: truth or fiction? J Immunother Cancer. 4: 48.

4. Schadendorf D, Wolchok JD, Hodi FS, Chiarion-Sileni V, Gonzalez R, et al (2017) Efficacy and Safety Outcomes in Patients With Advanced Melanoma Who Discontinued Treatment With Nivolumab and Ipilimumab Because of Adverse Events: A Pooled Analysis of Randomized Phase II and III Trials. J Clin Oncol. 35: 3807-14. [Crossref]

5. Schachter J, Ribas A, Long GV, Arance A, Grob JJ, et al. (2017) Pembrolizumab versus ipilimumab for advanced melanoma: final overall survival results of a multicentre, randomised, open-label phase 3 study (KEYNOTE-006). Lancet. 390: 1853-62. [Crossref]

6. Robert C, Ribas A, Hamid O, Daud A, Wolchok JD, et al. (2017) Durable Complete Response After Discontinuation of Pembrolizumab in Patients With Metastatic Melanoma. J Clin Oncol. Jco2017756270. [Crossref]

7. Schumacher TN,Schreiber RD. (2015) Neoantigens in cancer immunotherapy. Science. 348: 69-74.

8. Van Allen EM, Miao D, Schilling B, Shukla SA, Blank C, et al. (2015) Genomic correlates of response to CTLA-4 blockade in metastatic melanoma. Science. 350: 207-11. [Crossref]

9. Rizvi NA, Hellmann MD, Snyder A, Kvistborg P, Makarov V, et al. (2015) Cancer immunology. Mutational landscape determines sensitivity to PD-1 blockade in nonsmall cell lung cancer. Science. 348: 124-8. [Crossref]

10. Powles T, Eder JP, Fine GD, Braiteh FS, Loriot Y, et al. (2014) MPDL3280A (antiPD-L1) treatment leads to clinical activity in metastatic bladder cancer. Nature. 515: 558-62. [Crossref]

11. Johnson BE, Mazor T, Hong C, Barnes M, Aihara K, et al. (2014) Mutationa analysis reveals the origin and therapy-driven evolution of recurrent glioma. Science. 343: 189-93. [Crossref]

12. Padovan-Merhar OM, Raman P, Ostrovnaya I, Kalletla K, Rubnitz KR, et al. (2016) Enrichment of Targetable Mutations in the Relapsed Neuroblastoma Genome. PLoS Genet. 12: e1006501. [Crossref]
Citation:

Zin Myint, Ruta Arays, Arun K. Arumugam Raajasekar, Peng Wang (2018) Long-Term Outcomes in Patients after Discontinuation of Immune Checkpoint Inhibitors. J Clin Res Med Volume 1(3): 1-4 